\title{
BALLODORA MARCELI N. SP., VORTICELLIDE COMMENSAL DU CRUSTACÉ ISOPODE TERRESTRE TRICHONISCUS (SPILONISCUS) PROVISORIUS RACOVITZA
}

\author{
Par Paul REMY \\ Assistant à la Faculté des Sciences de Nancy
}

Les infusoires, êtres essentiellement aquatiques, se rencontrent parfois à la surface du corps d'animaux aériens mais, là encore, ils continuent à mener une existence aquatique : ils sont localisés dans une pellicule liquide qui recourre les téguments de leur hôte. Ainsi l'hétérotriche Conchophthirus steenstrupi Stein vit dans le mucus de la surface du corps de divers gastropodes pulmonés terrestres: Arion, Limax, Helix, Clausilia et surtout Succinea ; Cothurnia ligiæ Cuénot, fixé sur les lamelles branchiales de Ligia oceanica Fabr., est immergé dans la mince couche aqueuse qui humecte ces appendices.

Ces années dernières, j’ai rencontré en abondance des vorticellides très proches des Opercularia sur les lamelles branchiales de divers crustacés isopodes terrestres provenant de l'est de la France: Armadillidium vulgare Latr., Porcellio scaber Latr., Cylisticus convexus de Geer, Oniscus asellus L., Trichoniscus (Spiloniscus) provisorius Racovitza, Tr. (Androniscus) dentiger Verhoeff (1). J'ai rencontré également ces infusoires sur les pléopodes d'Oniscus asellus que j'ai récoltés sur l'île Lewis (Hébrides) au cours de la croisière aretique faite en 1926 par le Pourquoi-Pas ? sous la direction du Commandant Charcot. J'avais commencé l'étude des formes qui vivent sur les Porcellio et les Oniscus quand, en septembre 1927, M. le professeur Dubosq a eu l'obligeance de me signaler que de tels vorticellides avaient été rencontrés en Russie en 1920 sur les lamelles branchiales de Porcellio sp. ; leur étude avait été faite par Dogiel et Furssenko (1921) qui ont classé ces infusoires dans le nouveau genre Ballodora, proche du genre Opercularia, et les ont nommés $B$. dimorpha n. sp. J'ai reconnu alors que les vorticellides des Porcellio scaber que j'ai examinés appartiennent à cette espèce ; de celle-ci, je ne suis pas encore parvenu à distinguer

(1) Les deux Trichoniscus ont été déterminés par M. A. Vandel, Professeur à l'Université de Toulouse.

Annales de Parasitologie, T. VI, $\mathrm{N}^{\circ} 4 .-1^{\text {er }}$ octobre 1928, p. 419-430. 
les commensaux des Oniscus asellus de France et des Hébrides, et je les rapporte provisoirement à cette forme, qui aurait ainsi une distribution géographique étendue.

Je décris dans cette note un Ballodora nouveau, vivant sur certains pléopodes de Trichoniscus provisorius récoltés près de Servance (Haute-Saône) et aux environs de Nancy; en mémoire de mon frère, je l'appelle $B$. marceli.

Chez $T r$. provisorius, chaque pléopode est formé, comme chez tous les isopodes, de deux pièces, exopodite et endopodite, articulées sur un sympodite; chez les Trichoniscus des deux sexes, les exopodites I à V sont des lames rigides, recouvertes d'une assise chitineuse calcifiée relativement épaisse ; les endopodites III, IV et $\mathrm{V}$ sont des lamelles molles, recouvertes d'une couche de chitine excessivement mince, peu ou pas calcifiée, tandis que les endopodites I et II sont très chitineux, rigides et transformés plus ou moins profondément suivant le sexe (1). Les pléopodes sont humectés par un liquide incolore, visqueux, très réfringent, qui est sécrété par des glandes cutanées [v. Collinge (1921)].

Je n'ai rencontré $B$. marceli que sur les endopodites III à V. Les Trichoniscus de grande taille qui ont mué depuis quelque temps sont infestées dans la proportion de 8 p. 100 à Servance, de 75 p. 100 près de Tomblaine (environs de Nancy); cette proportion est beaucoup plus faible chez les adultes qui ont mué depuis peu et chez les jeunes. Tous les $T$. provisorius de ces deux stations étaient des femelles; on sait en effet [Vandel (1923).] que, dans l'Europe septentrionale et moyenne, les mâles de cet Isopode sont excessivement rares, l'espèce se multipliant là, en règle générale, par parthénogenèse. A une station voisine de Jarville (banlieue de Nancy), j'ai trouvé, fait exceptionnel, une forte proportion de mâles (30 sur . 67 exemplaires, examinés par M. Vandel) ; de cette station, j'ai disséqué 18 individus de grande taille : 11 femelles et 7 mâles ; 7 femelles étaient infestées, ce qui est la proportion normale, et tous les màles étaient indemnes. Mais, étant donné le petit nombre d'individus examinés, on ne peut conclure que tous les mâles de cette station sont toujours exempts de vorticellides (2).

Chez les Porcellio et les Osniscus, B. dimorpha est fixé également sur les endopodites des pléopodes III à V (3); ce commensal est

(1) Voir la description de ces pléopodes donnée par Racovilza (1908-1909, p. 257). J'ai çonstaté que les exopodites 1 à $V$ et les endopodites III à $V$ sont comme chez Oniscus a sellus L., Philoscia muscorum Scop. [Remy (1925, p. 124)], les Porcellio, etc., des organes respiratoires.

(2) A noter que les Porcellio et Oniscus des deux sexes portent des Ballodora.

(3) Dogiel et Furssenko en ont rencontré aussi des individus isolés sur les " couvercles » (exopodites) des Porcellio; je n'ai pu faire semblable observation. 
souvent beaucoup moins abondant sur les endopodites $\mathrm{V}$ que sur ceux des deux paires précédentes, sans doute parce que les pléopodes postérieurs sont moins humectés que les autres; chez les Trichoniscus, les endopodites III à V semblent être tous également infestés et les commensaux peuvent être rencontrés sur toute leur étendue ; souvent cependant ils sont plus abondants sur la face postérieure que sur l'autre; ils sont particulièrement nombreux dans la région basilaire, qui est la moins exposée à la dessiccation.

Pourquoi les Ballodora, aussi bien ceux des Porcellio et des Oniscus que ceux des Trichoniscus, sont-ils localisés sur les seules lames pléopodiennes qui soient revêtues d'une mince couche de chitine? Cette localisation semble être liée au facteur nourriture : ces infusoires se nourrissent de bactéries bacilliformes, rectilignes ou légèrement incurvées, longues de $0 \mu, 5$ à $5 \mu$, quelquefois libres dans le liquide qui humecte les endopodites III à V, mais le plus souvent fixées par une extrémité sur ces lamelles, formant une sorte de brosse parfois très dense (fig. 2, II, b) ; quelquefois, parmi ces bâtonnets, sont implantées des bactéries filamenteuses plus ou moins arquées dont la longueur peut dépasser $10 \mu$ (1).

Ballodora marceli présente, comme $B$. dimorpha, des individus sédentaires et des individus migrateurs.

I. Individus sédentaires. - Epanouis, ils ont la forme d'une gourde ovoïde dont le goulot, large et court, correspond à la collerette. Souvent le corps de la gourde est légèrement asymétrique ; une face latérale étant moins convexe que les autres (fig. 1), Certains individus sont de grande taille : $50 \mu$ de long sur $40 \mu$ de large; d'autres ont des dimensions beaucoup plus réduites : $30 \mu$ de long sur 25 de large ; entre ces deux types extrêmes, on observe de nombreuses formes de passage.

a) Grands individus (fig. 1). - Ils sont portés par un pédicule non contractile, court et épais (sa longueur ne dépasse guère $8 \mu$ et la largeur de sa partie moyenne $3 \mu$ ); l'extrémité du pédicule qui est en contact avec la surface de l'endopodite s'élargit assez brusquement, formant une surface d'adhésion à contour circulaire ; l'autre extrémité se dilate progressivement en formant un tronc de cône dont la grande base est en contact avec le corps de l'animal ; à cet endroit se trouve la bordure en brosse circulaire qui a été décrite chez divers vorticellides par Fauré-Fremiet (1905)

(1) Ce mode de fixation est analogue à celui que présentent, à la surface de l'épithélium intestinal, certaines bactéries parasites du tube digestif d'insectes : bacilles chez les larves de chironome et d'éristale, spirochète chez les larves de chironome (Léger), bactérie filamenteuse chez le grillon domestique (Léger et Duboscq) [v. Léger (1902)]. 
sous le nom de scopula et au niveau de laquelle est sécrété le pédicule. Celui-ci présente une striation longitudinale très nette due à un faisceau de tigelles squelettiques analogue à celui qu'on trouve dans le pédicule des Opercularia; ce faisceau, bien visible après coloration par le rouge congo, s'épanouit dans la région tronconique voisine de la scopula. Lorsque l'animal est contracté, la

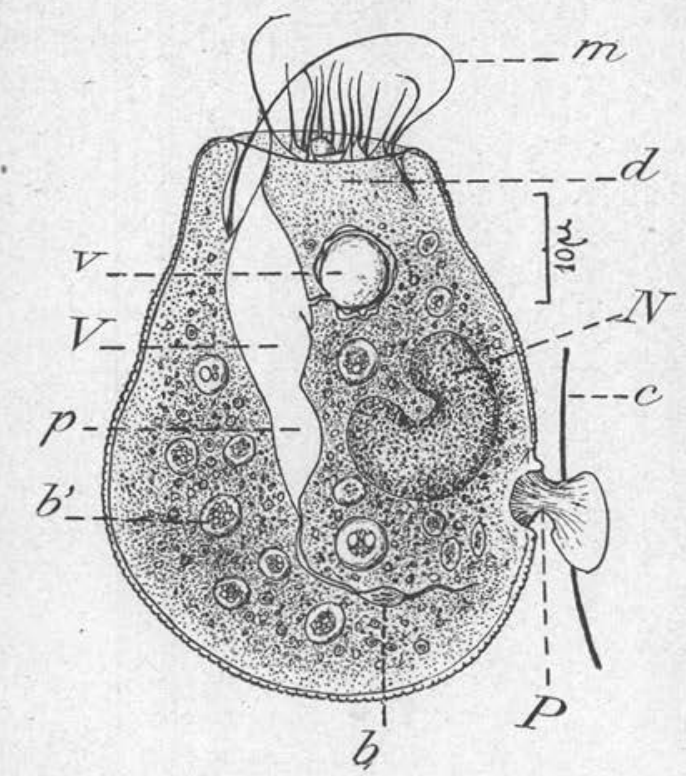

FIg. 1. - Ballodora marceli. Grand individu sédentaire, vivant, épanoui : $b, \quad b$, bols alimentaires; $c$, contour de l'endopodite qui porte l'infusoire ; $d$, disque ; $m$, membrane ondulante ; $N$, macronucleus ; $P$, pédicule ; $p$, pharynx ; $v$, vésicule pulsatile, entourée de son boyau sinueux : V, vestibule. (La striation de la cuticule n'est indiquée que sur les bords; les bactéries fixées sur l'endopodite ne sont pas figurées).

partie postérieure du corps forme un bourrelet circulaire qui recouvre presque toute l'étendue du pédicule.

Chez les individus asymétriques (fig. 1), le pédicule ne s'insère pas sur le pôle aboral, comme c'est la règle chez les vorticellides, mais sur la face aplatie de l'animal, à une certaine distance de ce pôle ; l'axe du corps est alors perpendiculaire à celui du pédicule, de sorte que l'infusoire est nettement couché sur le côté, sa face latérale aplatie étant en contact avec la surface de l'endopodite.

Aplatissement de ces gros individus, faible longueur de leur pédicule, insertion de celui-ci sur le côté de l'animal, tous ces caractères 
représentent des adaptations au milieu si spécial dans lequel vivent ces vorticellides : ils permettent aux Ballodora de s'immerger complètement dans la pellicule liquide qui recouvre les endopodites.

Le corps est revêtu d'une cuticule incolore, striée transversalement, relativement épaisse (3/4 de $\mu$ environ), résistant à l'action de l'acide acétique glacial; un tégument épais se rencontre chez tous les Opercularia commensaux d'animaux aquatiques; il oppose une certaine résistance aux pressions exercées sur l’infusoire, soit

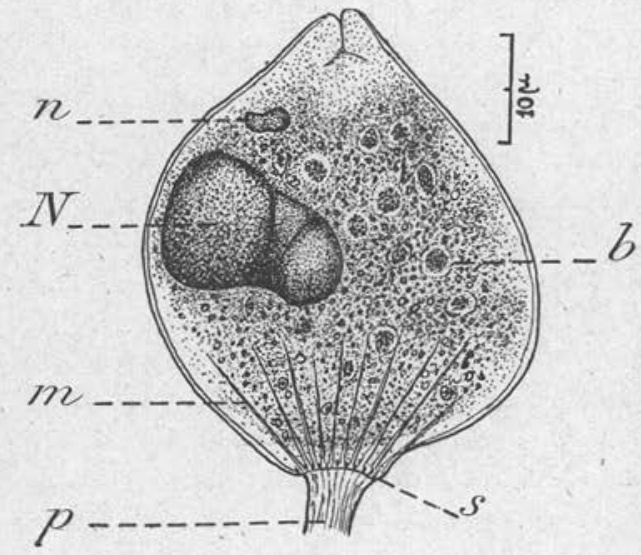

I

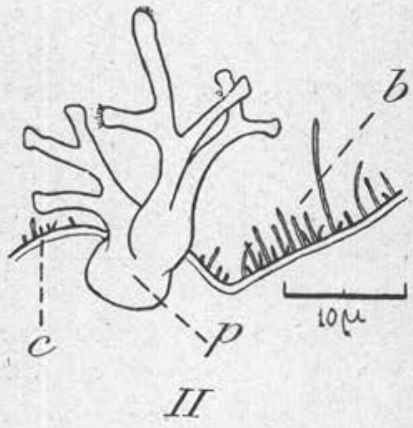

Fig. 2. - Ballodora marceli. I. Individu sédentaire résultant de la bipartition d'un gros exemplaire; coloration à l'acétocasmin au $\mathrm{f}(\mathrm{r}$ : $b$, bol alimentaire ; $m$, myonème; $n$, micronucleus ; $N$, macronucleus ; $p$, pédicule ; $s$, scopula. (La striation de la cuticule n'est pas figurée). II. Pédicule $p$, d'une colonie dont 2 individus migrateurs se sont détachés (à l'extrémité de leur rameau pédiculaire dépassent quelques tigelles squelettiques); les autres individus n'ont pas été représentés; $c$, cuticule de l'endopodite; elle porte des bactéries $b$ formant une brosse. Coloration au rouge congo.

par l'eau ambiante quand l'hôte se déplace (cas des Opercularia), soit par les lames pléopodiennes de l'isopode (cas de Ballodora); de plus, il protège le protozoaire contre la dessiccation (1).

Le disque (fig. $1, d$ ), de petite taille, rappelle assez celui que Fauré-Fremiet (1905) a décrit chez un petit groupe d'Opercularia distingué par cet auteur sous le nom de Cochlearia; cylindrique dans sa partie inférieure, il se dilate dans sa région supérieure, surtout sur la face qui limite l'entrée du vestibule, puis il se rétrécit

(1) Opercularia notoneclae Fauré-Fremiet, commensal de Notonecta glauca L., est pourvu d'un tégument épais, à structure complexe, et peut parfaitement résister à une dessiccation temporaire lorsque l'insecte sort de l'eau. 
brusquement et se termine par un petit mamelon hémisphérique axial dont le sommet est à peu près au niveau des bords de la collerette. Autour du disque, la frange adorale décrit une hélice de deux tours et demi environ; cette frange ne vibre, pendant les périodes d'extension, que par intermittences, en général un peu avant que le disque ne se rétracte : les temps de pose en étalement rigide sont relativement longs.

La collerette, comme chez les Opercularia, ne se replie jamais en dehors; quand l'animal se rétracte, elle se ferme par simple rapprochement de ses bords; vue de face, son ouverture virtuelle a alors la forme d'une fente à lèvres plissées.

Le vestibule (fig. $1, \mathrm{~V}$ ), rétréci à son entrée par la partie supérieure, élargie, du disque, se dilate dans sa partie moyenne, puis son diamètre diminue progressivement jusqu'à l'endroit où s'ouvre le pharynx $p$. Celui-ci, légèrement renflé dans sa région moyenne, est effilé à son extrémité postérieure et se continue par un canal long et étroit, fortement arqué (canal pharyngien de Fauré-Fremiet), qu'on peut suivre grâce au cheminement à son intérieur des bols alimentaires fusiformes $b$.

Alors que l'appareil pulsatile des infusoires commensaux, et surtout celui des infusoires parasites, sont en général réduits, celui des $B$. marceli est bien développé. La vésicule $v$, souvent irrégulière quand elle commence à se remplir, prend par la suite la forme d'une sphère dont le diamètre atteint $6 \mu$ quand elle est sur le point de se vider ; son contenu est déversé directement dans le vestibule, par un petit conduit. Dogiel et Furssenko ont constaté que, chez $B$. dimorpha, la vésicule pulsatile fonctionne très activement: ils ont compté 6 à 8 systoles par minute (examen dans l'eau), tandis que chez la majorité des infusoires, il n'y en a que 2 ou 3 ; chez $B$. marceli, il s'écoule de 15 à 22 secondes entre deux systoles consécutives, ce qui correspond à 3 ou 4 pulsations par minute (examen dans l'eau distillée).

La vésicule pulsatile est entourée par une formation très curieuse, qui est tout à fait analogue à celle que Dogiel et Furssenko ont décrite chez $B$. dimorpha, et qui n'a pas encore été rencontrée chez les autres péritriches. Cette formation a l'aspect d'un boyau irrégulièrement sinueux, réfringent, dont la largeur varie le long de son étendue : le diamètre de certains segments n'est que de quelques dixièmes de $\mu$, tandis qu'ailleurs, il atteint $3 / 4$ de $\mu$. Je ne peux affirmer que ce cordon forme un anneau complet autour de la vésicule ; lorsque celle-ci se vide, le boyau se chiffonne, ses sinuosités sont plus nombreuses et plus accentuées, mais il n'est pas certain qu'il se contracte d'une façon active : peut-être suit-il 
passivement les contours de la vésicule; ses ondulations s'effacent de plus en plus à mesure que la vacuole se remplit à nouveau. La structure et la fonction de cette singulière formation demeurent problématiques : Dogiel et Furssenko pensent que, selon toute vraisemblance, le boyau " aide la vacuole, d'une façon ou d'une autre, à vider son contenu ». Jouerait-il le rôle de canal collecteur du liquide vésiculaire, canal qui se remplirait pendant que la vacuole se vide, et vice-versa? Il ne m'a pas semblé que le diamètre d'un segment donné du boyau varie avec l'état de la vésicule. Je crois cependant que cette formation intervient dans l'élaboration du liquide vésiculaire : c'est toujours au contact d'un de ses segments élargis que la vésicule commence à se remplir; plusieurs fois j'ai vu deux petites vésicules se former simultanément, chacune étant au contact d'un de ces larges segments (en grossissant, ces vésicules se sont fusionnées).

Le cytoplasme est très finement 'granuleux dans le disque, dans la région périphérique du corps et dans une zone en forme de croissant située immédiatement au-dessus de la scopula ; ailleurs, il renferme de grosses granulations intercalées entre les bols alimentaires. Ceux-ci, lorsqu'ils sont formés depuis peu, renferment des bactéries ; âgés, ils contiennent des granules incolores ou brun plus ou moins foncé.

Le macronucléus de $B$. marceli est très différent de celui de $B$. dimorpha. Ce dernier est un cordon grêle, courbé en forme de fer à cheval, de 6 ou d'S (dont les 2 anses sont souvent situées dans deux plans perpendiculaires); sa longueur, qui est en général de 55 à $60 \mu$, peut atteindre $75 \mu$; sa largeur varie de 6 à $8 \mu$. Celui de $B$. marceli est plus court, plus trapu : sa longueur dépasse rarement $20 \mu$, sa largeur atteint 6 à $8 \mu$; son incurvation, légère dans la partie moyenne, s'accentue dans les régions terminales de sorte que le noyau a la forme d'un anneau elliptique auquel manquerait la portion située à l'une des extrémités du petit axe ; parfois l'incurvation est moins prononcée, et le noyau a la forme d'un saucisson, ou bien ses deux moitiés s'accolent par leur face concave et il prend une forme globuleuse ; chez certains individus, il présente un court diverticule latéral, analogue à celui que Dogiel et Furssenko ont représenté dans leur fig. 6 chez $B$. dimorpha.

Le micronucléus de $B$. dimorpha est un corpuscule oblong, de $3 \mu$ à $3 \mu, 4$ de long sur $1 \mu, 6$ à $2 \mu$ de large, situé en général à quelque distance (jusqu'à 15 ou $20 \mu$ ) du macronucléus, le plus souvent dans la région antérieure du corps; on le met facilement en évidence avec l'acéto-carmin au fer de Belling (1921). Chez B. marceli, le micronucléus est généralement accolé au macronucléus et 
est difficilement visible ; chez certains individus, cependant, il peut s'écarter jusqu'à une distance d'une dizaine de $\mu$ du macronucléus ; long de 3 à $4 \mu$, large de $1 \mu, 5$ à $2 \mu$, il présente parfois une légère constriction en son milieu.

Un faisceau de myonèmes diverge de la partie inférieure de l'individu.

b) Petits individus. - Les gros individus sédentaires de B. marceli se multiplient par bipartition suivant un plan longitudinal qui

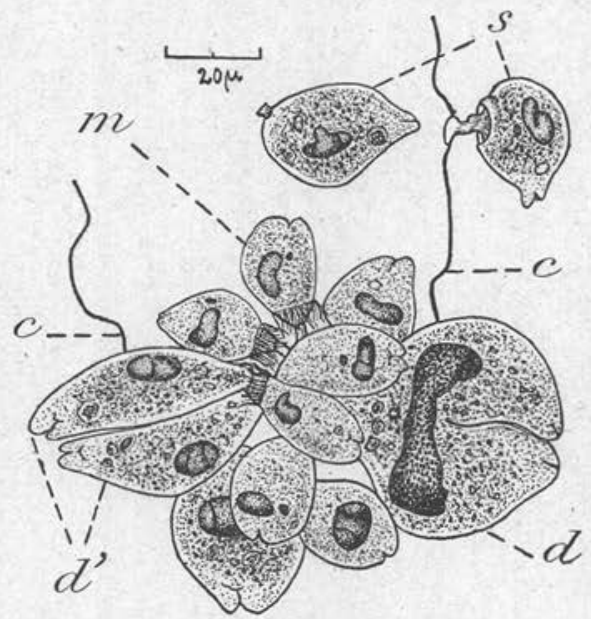

Fig. 3. - Colonie en rosette de Ballodora marceli fixée à l'extrémité distale de l'endopodite d'un pléopode $V$. Coloration à l'acêtocarmin au fer : $c$, contour de l'endopodite ; $d$, gros individu sédentaire en bipartition ; $d$ ', 2 de ces individus résultant d'une bipartition récente; $m$, individu migrateur; $s, 2$ petits individus sédentaires; chez celui de droite, le micronucleus est bien visible; l'autıe exemplaire a un macronucleus lobé latéralement. Les bactéries fixées sur l'endopodite ne sont pas figurées.

apparaît à l'extrémité libre de l'animal et progresse vers le pédicule (fig. $3, d$ et $d^{\prime}$ ) ; les deux individus qui résultent de cette division sont identiques. chacun d'eux secrète un pédicule qui apparaît comme une ramification du pédicule primitif. Les mêmes phénomènes s'observent quand ces deux individus se divisent à leur tour (1) ; les bipartitions répétées ont pour résultat la formation d'une colonie en rosette (fig. 3), dont les individus, qui peuvent être au nombre d'une douzaine, sont situés à l'extrémité des branches du pédicule initial, plusieurs fois ramifié (fig. 2, II). A noter que

(1) Ces deux divisions sont très rarement simultanées. 
les rameaux de la colonie sont peu inclinés sur la surface de l'endopodite, de sorte que les animaux demeurent immergés dans la pellicule liquide qui recouvre l'appendice.

La plupart des individus des grandes colonies ont une taille réduite et sont portés par des pédicules relativement longs pouvant atteindre $15 \mu$; ceux dont la taille est voisine de $45 \times 35 \mu$ (fig. 2, I), ont une structure analogue à celle des gros individus sédentaires décrits précédemment ; ceux qui sont plus petits renferment moins de bols alimentaires et leur cytoplasme est plus finement granuleux.
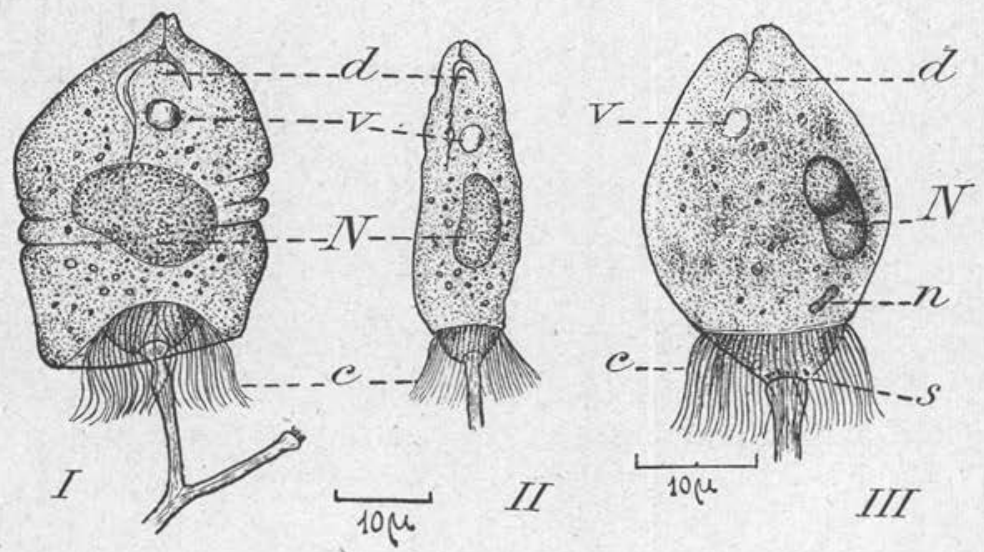

Fig. 4. - Individus migrateurs de Ballodora marceli. I, contracté; II, en extension (observés vivants); III, coloré par l'acétocarmin au fer ; $c$, couronne aborale ; $d$, disque (sur celui de I, la frange adorale est indistinctement visible); $n$, micronucleus; $N$, macronucleus; $s$, scopula; $v$, vésicule pulsatile. En I, l'individu frère s'est déjà détaché.

B. Individus migrateurs. - Ces petits individus sédentaires se transforment en individus migrateurs (fig. 4), mais il est rare que les deux individus frères se modifient simultanément. Le disque se rétracte et forme un mamelon conique, plus large que haut, demeurant constamment caché par les parois de la collerette qui se sont rapprochées en se plissant longitudinalement ; sur ce mamelon, on peut apercevoir l'hélice adorale dont les membranelles s'agitent quelquefois, mais faiblement.

Chez les individus les plus transformés, le corps, très étiré (longueur 35 à $45 \mu$, largeur 10 à $15 \mu$ ) lorsqu'il est en extension (fig. 4 , II), présente, dans sa région moyenne, de gros plis transversaux lorsqu'il est contracté (fig. $4, \mathrm{I}$ ) ; il se développe une couronne aborale (fig. $4, c)$, formée de cils relativement longs (10 à $15 \mu$ ), animés 
de mouvements ondulatoires très lents ; cette couronne est insérée au fond d'un petit sillon circulaire situé à une distance de la scopula égale à $1 / 8$ environ de la longueur du corps en extension. Lorsque l'animal est contracté (fig. 4, I), la partie du corps située entre le pédicule et la ceinture locomotrice s'invagine ; cette dernière est alors insérée au fond d'un profond repli.

Le vestibule et le pharynx sont difficilement visibles; les membranes ondulantes s'agitent parfois, mais faiblement; les bols alimentaires ont disparu totalement.

La vésicule pulsatile persiste mais ne se vide plus que toutes les 45 secondes environ; son diamètre maximum est réduit $(2 \mu, 5$ à $4 u$ ) ; je n'ai pu apercevoir de formation en boyau autour d'elle.

Le macronucléus (fig. 4, III, $N$ ), gros, court, est arqué en fer à cheval plus ou moins ouvert ; le micronucléus (fig. 4 , III, $n$ ), presque toujours très facilement visible après coloration par l'acétocarmin au fer, est généralement situé à quelque distance du macronucléus.

A un certain moment, ces individus agitent activement leur couronne aborale et, prenant appui avec leur corps sur l'endopodite ou sur les gros individus sédentaires voisins, ils se détachent de leur pédoncule au niveau de la scopula. L'animal nage alors librement en tournant autour de son axe qùi est lui-même incliné sur la trajectoire. Je n'ai pu suivre le sort de ces individus libres.

De telles formes migratrices apparaissent fréquemment chez les vorticellides coloniaux ; après s'être-détachées, elles vont se fixer ailleurs, perdent leur ceinture locomotrice, sécrètent un pédicule et prennent un appareil adoral normal; chacune constitue alors le premier individu d'une colonie nouvelle. Les petits Ballodora sédentaires isolés que l'on rencontre assez souvent sur les lamelles branchiales des Trichoniscus (fig. 3, en haut), sont très probablement des individus migrateurs qui ont subi une telle modification. Ce sont ces formes mobiles qui doivent propager l'espèce non seulement sur le corps de leur hôte au moment de la mue et entre les mues, mais aussi sur celui des Trichoniscus voisins, probablement en nageant dans l'eau qui imbibe les détritus dans lesquels vivent ces isopodes : j'ai vu un de ces individus se déplacer pendant plus d'une heure et demie dans l'eau.

Les conjugaisons sont très rares chez les Ballodora. Dogiel et Furssenko en ont rencontré « deux ou trois fois seulement, et chez les individus isolés 》 de $B$. dimorpha; j'en ai observé une chez cette espèce et une chez un Ballodora nouveau que j'ai trouvé sur les lamelles branchiales de Trichoniscus (Androniscus) dentiger Verhoeff, provenant de Nancy. Ces conjugaisons sont anisogami- 
ques ; dans les deux cas que j'ai observés, le macrogamète était un gros individu sédentaire isolé ; le microgamète, dont la taille correspond à celle des individus mobiles, était accolé par son pôle aboral au niveau du tiers postérieur de son conjoint et était dépourvu de ceinture locomotrice.

Un problème intéressant se pose : que deviennent les commensaux de l'isopode lorsque celui-ci mue ? Comment les lamelles branchiales qui viennent de muer se peuplent-elles à nouveau en infusoires? Dogiel et Furssenko l'ont résolu chez B. dimorpha: les individus mobiles quittent l'exuvie et vont se fixer sur la nouvelle cuticule ; en outre, les gros individus sédentaires isolés muent en même temps que l'endopodite qui les porte : leur corps se détache de la pellicule, passe d'abord à l'intérieur du pédicule (qui est devenu creux) puis à travers un orifice percé dans l'exuvie de l'endopodite au point d'insertion du pédicule; l'animal se fixe alors sur la nouvelle cuticule. Je n'ai pas eu la chance de rencontrer un isopode en état de mue convenable et n'ai pu refaire ces très intéressantes observations.

Les Ballodora présentent de très grandes affinités avec les Opercularia, dont certaines espèces vivent fixées sur les végétaux ou les détritus immergés tandis que d'autres sont commensales de nombreux arthropodes aquatiques, en particulier de crustacés. Sont-ils les ancêtres ou les descendants des Opercularia? Si l'on accepte la seconde supposition, sont-ils issus d'Opercularia non commensaux ou bien, comme on serait plus tenté de le croire, proviennent-ils d'hypothétiques Opercularia qui auraient vécu sur les ancêtres aquatiques des isopodes terrestres actuels et qui se seraient modifiés lorsque ces crustacés ont quitté l'eau ? Autant de questions auxquelles il serait prématuré de vouloir répondre d'une façon sérieuse actuellement.

\section{BiBLIOGRAPHIE}

Belling (J.). - On counting chromosomes in pollen-mother cells. Amer. Nat., LV, 1921, p. 573-574.

Collinge (W.-E.). - A preliminary study of the structure and function of the cutaneous glands in the terrestrial Isopoda. Ann. Mag. Nat. Hist., [9], VII, 1921, p. 212-222.

Cúx́ot (L.). - Infusoires commensaux des Ligies, Patelles et Arénicoles. Rev. biol. Nord de la Fr., IV, 1891-1892, p. 81-89.

Dogiel (V.) et Furssenko (A.). - Neue ektoparasitische Infusorien von Landisopoden. Trav. Soc. Nat. Pelrograd, Sect. 1 C. R., LI, 1921, p. 147-158 (en russe ; rés. allem., p. 199-202). 
Fauré- Fremiet (E.). - Note sur un nouveau groupe d'Opercularia. Arch. Anat. micr., VII, 1905, p. 181-197.

Fauré-Fremiet (E.). - La structure de l'appareil fixateur chez les Vorlicellida. Arch. Protistenk., VI, 1905, p. 207-226.

LÉger (L.). - Bactéries parasites de l'intestin des larves de Chironone. $C$. $R$. Acad. Sc., CXXXIV, 1902, p. 1317-1319.

Racovitza (E.-G.). - Isopodes terrestres (seconde série). Biospeologica IX. Arch. zool. exp., [4], IX, 1908-1909, p. 239-415.

REMY (P.). - Contribution à l'étude de l'appareil respiratoire et de la respiration chez quelques Invertébrés. Th. Sc. Nancy, 1925, 222. p.

VANDEL (A.). - L'existence et les conditions de la parthénogenèse chez un Isopode terrestre: Trichoniscus (Spiloniscus) provisorius Racovitza. C. R. Acad. Sc., CLXXVII, 1923, p. 793-795. 